\title{
Automatic calculation of the magnetometer zero offset using the interplanetary magnetic field based on the Wang-Pan method
}

\author{
XiaoWen Hu' ${ }^{1}$, GuoQiang Wang ${ }^{2 *}$, and ZongHao Pan ${ }^{1}$ \\ ${ }^{1}$ Chinese Academy of Sciences Key Laboratory of Geospace Environment, School of Earth and Space Sciences, University of Science and Technology of China, \\ Hefei 230026, China; \\ ${ }^{2}$ Institute of Space Science and Applied Technology, Harbin Institute of Technology, Shenzhen 518055, China \\ Key Points: \\ - We propose an automatic procedure to calculate the zero offset of the space-borne FGM using the data in the solar wind. \\ - The procedure can automatically select potential Alfvénic fluctuation events, obtain their optimal offset lines, and then calculate the \\ zero offset. \\ - The test results based on the Venus Express satellite data show that our method can easily and intuitively obtain the zero offset.
}

Citation: Hu, X. W., Wang, G. Q., and Pan, Z. H (2022). Automatic calculation of the magnetometer zero offset using the interplanetary magnetic field based on the Wang-Pan method. Earth Planet. Phys., 6(1), 52-60. http://doi.org/10.26464/epp2022017

\begin{abstract}
The space-borne fluxgate magnetometer (FGM) requires regular in-flight calibration to obtain its zero offset. Recently, Wang GQ and Pan ZH (2021a) developed a new method for the zero offset calibration based on the properties of Alfvén waves. They found that an optimal offset line (OOL) exists in the offset cube for a pure Alfvén wave and that the zero offset can be determined by the intersection of at least two nonparallel OOLs. Because no pure Alfvén waves exist in the interplanetary magnetic field, calculation of the zero offset relies on the selection of highly Alfvénic fluctuation events. Here, we propose an automatic procedure to find highly Alfvénic fluctuations in the solar wind and calculate the zero offset. This procedure includes three parts: (1) selecting potential Alfvénic fluctuation events, (2) obtaining the $\mathrm{OOL}$, and (3) determining the zero offset. We tested our automatic procedure by applying it to the magnetic field data measured by the FGM onboard the Venus Express. The tests revealed that our automatic procedure was able to achieve results as good as those determined by the Davis-Smith method. One advantage of our procedure is that the selection criteria and the process for selecting the highly Alfvénic fluctuation events are simpler. Our automatic procedure could also be applied to find fluctuation events for the Davis-Smith method.
\end{abstract}

Keywords: fluxgate magnetometer; in-flight calibration; zero offset; highly Alfvénic fluctuations; automatic procedure

\section{Introduction}

Dynamic processes, such as reconnections (Zhang TL et al., 2012; Lu S et al., 2020), instabilities (Hellinger et al., 2017; Duan AY et al., 2018), turbulences (Huang SY et al., 2018; Xiao SD et al., 2020a, b), linear magnetic holes (Ge YS et al., 2011; Wang GQ et al., 2020a), and plasma waves (Keiling, 2009; Wang GQ et al., 2015, 2016, 2017; Sun JC et al., 2019, 2020, 2022), are abundant in the space plasma environment. Some kinetic-scale processes or structures require accurate measurement of the magnetic field (Burch et al., 2016; Wang GQ et al., 2021b). Therefore, high-precision measurement of the magnetic field is crucial to investigate the physical processes in space.

The fluxgate magnetometer (FGM) is one of the most widely used instruments for detecting the magnetic field in space (Acuña, 2002; Balogh, 2010; Burch et al., 2016; Liu K et al., 2020). To accur-

Correspondence to: G. Q. Wang, wanggq@hit.edu.cn

Received 24 NOV 2021; Accepted 24 DEC 2021.

Accepted article online 13 JAN 2022.

C) 2022 by Earth and Planetary Physics. ately measure the magnetic field, the FGM needs to be calibrated before launch of the spacecraft (Olsen et al., 2003; Risbo et al., 2003). Nevertheless, performing regular in-flight calibration is still needed because its instrumental offset, the value measured in a null field environment, varies slowly with time (Olsen et al., 2003; Balogh, 2010). In addition, the slowly changing (or static) magnetic field generated by the spacecraft at the sensor position is generally not negligible, and the static magnetic field is difficult to distinguish from the instrumental offset (Pope et al., 2011; Pudney et al., 2012). Thus, both the static magnetic field and the instrumental offset are regarded as the zero offset of the spaceborne FGM (Leinweber et al., 2008). Alfvén waves (Davis and Smith, 1968; Belcher, 1973; Hedgecock, 1975) as well as mirror mode structures (Plaschke and Narita, 2016; Plaschke et al., 2017; Plaschke, 2019; Schmid et al., 2020; Wang GQ and Pan ZH, 2021b) can be used to calculate the zero offset.

The Davis-Smith method (Davis and Smith, 1968), the Belcher method (Belcher, 1973), and the Hedgecock method (Hedgecock, 1975) have been proposed to calculate the zero offset based on the properties of Alfvén waves. Both the Belcher method and the 
Hedgecock method require a long time interval (a few days or longer) of input data (Belcher, 1973; Hedgecock, 1975; Leinweber et al., 2008), which makes both methods unsuitable for in-flight calibration of a spacecraft partially orbiting in the solar wind, such as the Venus Express (VEX; Zhang TL et al., 2006) and the Magnetospheric Multiscale (MMS) Mission spacecrafts (Russell et al., 2016). Leinweber et al. (2008) found that the Davis-Smith method is mathematically superior to the other two methods and requires a much shorter time interval of input data. The accuracy of the zero offset calculation depends on the selection of the interplanetary magnetic field (IMF) fluctuations (Meng LF et al., 2018; Pan ZH et al., 2019; Wang GQ et al., 2019, 2020b).

Recently, Wang GQ and Pan ZH (2021a) have proposed a new method to calculate the zero offset of the FGM based on properties of the Alfvén wave. For the convenience of description, we refer to this new method as the Wang-Pan method. Wang GQ and Pan ZH (2021a) found that the zero offset is on a straight line determined by an Alfvén wave event in the offset cube, and they defined this line as the optimal offset line (OOL). They also found that the intersection of at least two nonparallel OOLs determined by different Alfvén waves is the zero offset. The Wang-Pan method can deal with the IMF fluctuation with a duration of less than 1 minute, and it calculates the zero offset more intuitively than the other methods (Wang GQ and Pan ZH, 2021a). Because no pure Alfvén waves exist in the IMF (see Leinweber et al., 2008), the selection of fluctuation events for the Wang-Pan method is expected to be critical. However, Wang GQ and Pan ZH (2021a) did not provide a method or criterion by which to select IMF fluctuations with highly Alfvénic nature.

In this study, we developed an automatic procedure to search out highly Alfvénic IMF fluctuations and then calculate the zero offset based on the Wang-Pan method. We first briefly introduce the Wang-Pan method in Section 2. We then provide a detailed description of the automatic procedure in Section 3. In Section 4, we apply this automatic procedure to perform the in-flight calibration of the FGM onboard the VEX spacecraft. Section 5 presents a summary of our work.

\section{Wang-Pan Method}

Alfvén waves do not change the magnetic field strength (Keiling, 2009). Wang GQ and Pan ZH (2021a) found, based on this property of Alfvén waves, that an OOL with good linearity exists in the offset cube and that this line passes through the real zero offset. The range of each side of the offset cube can be set according to the possible range of the IMF strength, which is typically less than $20 \mathrm{nT}$. The intersection of at least two nonparallel OOLs resulting from different Alfvén waves is expected to be the zero offset.

\subsection{Optimal Offset Line}

Here we introduce the definition of the OOL and show how to obtain the OOL of an Alfvén wave in the offset cube (Wang GQ and Pan ZH, 2021a). We assume that the sensitivities and non-orthogonal angles of the FGM have been calibrated except for the zero offset $\boldsymbol{O}\left(=\left(O_{X}, O_{Y}, O_{Z}\right)\right)$. The magnetic field data $B_{M}\left(=\left(B_{M} X_{1}, B_{M} Y_{\text {, }}\right.\right.$ $\left.B_{M_{\_} Z}\right)$ ) are thereby composed of only the natural magnetic field $\boldsymbol{B}_{A}\left(=\left(B_{A \_X}, B_{A_{-} Y}, B_{A \_Z}\right)\right)$ and $\boldsymbol{O}$, or $\boldsymbol{B}_{M}=\boldsymbol{B}_{A}+\boldsymbol{O}$. Because the typical value of the IMF strength is $<20 \mathrm{nT}$, the three components of $O$ are expected to be in the range of $\left(\left\langle B_{M_{-} x}\right\rangle-20,\left\langle B_{M_{-} x}\right\rangle+20\right)$, $\left(\left\langle B_{M_{-} Y}\right\rangle-20,\left\langle B_{M_{-} Y}\right\rangle+20\right)$, and $\left(\left\langle B_{M_{-} Z}\right\rangle-20,\left\langle B_{M_{-} Z}\right\rangle+20\right) \mathrm{nT}$, respectively, where $\left\langle B_{M_{-}}\right\rangle,\left\langle B_{M_{-} Y}\right\rangle$, and $\left\langle B_{M_{-} z}\right\rangle$ are the average values of the three components of $B_{M}$. Thus, an offset cube in the same coordinate system as $\boldsymbol{B}_{M}$ can be built, and the three axes of this offset cube are in the ranges of $\left(\left\langle B_{M_{-} x}\right\rangle-20,\left\langle B_{M_{-} x}\right\rangle+20\right)$, $\left(\left\langle B_{M_{-} \gamma}\right\rangle-20,\left\langle B_{M_{-} \gamma}\right\rangle+20\right)$, and $\left(\left\langle B_{M_{-} z}\right\rangle-20,\left\langle B_{M_{-} z}\right\rangle+20\right) \mathrm{nT}$, respectively. One can expect that the zero offset is a certain point in this offset cube.

The magnetic field data are modified to be $\boldsymbol{B}_{M}^{\prime}=\boldsymbol{B}_{A}+\mathbf{O}-\boldsymbol{O}^{\prime}$ at point $\boldsymbol{O}^{\prime}$ in the offset cube. To determine which point in the offset cube is the zero offset, Wang GQ and Pan ZH (2021a) tried to find the point that was most likely to be the zero offset in each parallel plane. For a pure Alfvén wave, the standard deviation $\delta$ of $\left|\boldsymbol{B}_{M}^{\prime}\right|$ is zero when $\mathbf{O}=\mathbf{O}^{\prime}$, and $\delta$ is a nonzero value when $\mathbf{O} \neq \mathbf{O}^{\prime}$. Therefore, Wang GQ and Pan ZH (2021a) optimized the potential zero offset in each parallel plane so that the point was most likely to be the zero offset by minimizing the value of $\delta$ in the corresponding plane. They also found that these points in the corresponding parallel planes were approximately on a straight line, which was defined as the OOL, because any point on this line could be the zero offset. Furthermore, they found that the OOL was parallel to the vector $\left(\left\langle B_{A_{-} X}\right\rangle,\left\langle B_{A_{-} Y}\right\rangle,\left\langle B_{A_{-}} z\right\rangle\right)$, where $\left\langle B_{A_{-} X}\right\rangle,\left\langle B_{A_{-} Y}\right\rangle$, and $\left\langle B_{A_{-} z}\right\rangle$ are the averages of the three components of $B_{A}$, respectively.

The physical meaning of the OOL can be better understood in the mean field-aligned (MFA) coordinate system. In this coordinate system, the $z$-axis is parallel to the ambient magnetic field, and the fluctuation of the Alfvén wave is only in the $x-y$ plane. Thus, the strength of the magnetic field in the $x-y$ plane $\sqrt{B_{x}^{2}+B_{y}^{2}}$ is a constant (Keiling, 2009; Wang GQ et al., 2015). In the offset cube, the nonconstant value of the modified magnetic field strength in the $x-y$ plane is expected to be caused by the $x$ and $y$ components of $O-O^{\prime}$. Thus, in the offset cube, we can obtain the $x$ and $y$ components of the zero offset in the MFA system. However, the $z$ component of the zero offset can be any value. Thus, we cannot determine the zero offset based on only a single Alfvén wave. The most likely values of the zero offset in the MFA system form a straight line parallel to the $z$-axis, and this line is the OOL.

\subsection{Determination of the Zero Offset}

To find the zero offset $\boldsymbol{O}$, at least two nonparallel OOLs are necessary. As shown in Figure 1, the intersection of the three nonparallel OOLs is the zero offset because all the OOLs pass through point $O$ in the offset cube.

Even if the corresponding IMF fluctuation event has a highly Alfvénic nature, the $\mathrm{OOL}$ is usually not a straight line because of the influence of the compressional wave and the magnetic field noise. In any case, the point that minimizes $\delta$ in each plane can be fitted into a straight line, which is called the fitted optimal offset line (FOOL). The FOOL usually does not pass through the zero offset point, resulting in no common intersection for the nonparallel FOOLs in the offset cube. Therefore, Wang GQ and Pan ZH (2021a) optimized the zero offset so that the sum of the distances from the point in the offset cube to all the FOOLs determined by differ- 


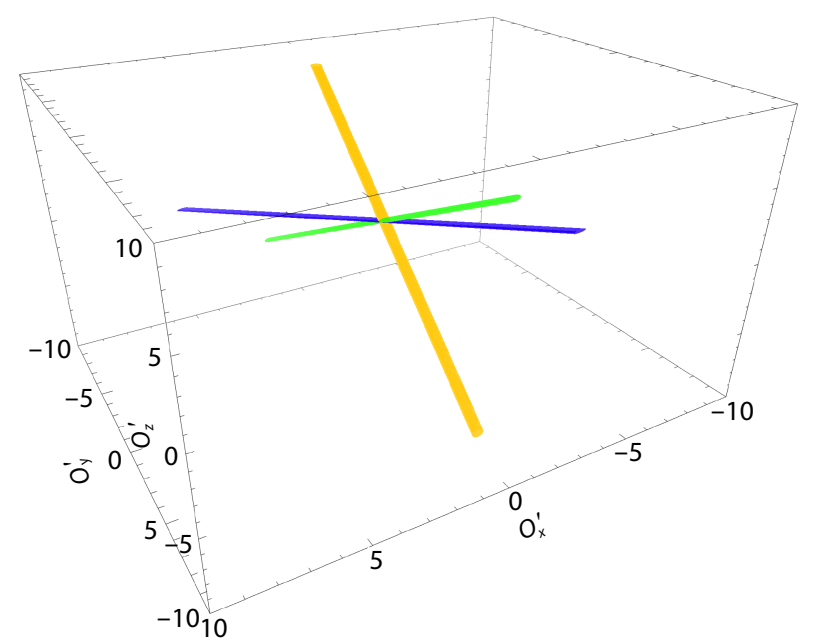

Figure 1. Schematic diagram of the zero offset determined by three nonparallel OOLs in the offset cube.

ent IMF fluctuation events was the smallest.

\section{Automatic Procedure}

For the Wang-Pan method, the process of determining the zero offset can be simplified as finding the point that minimizes the sum of the distances from this point to all the nonparallel FOOLs (Wang GQ and Pan ZH, 2021a). It is easy to obtain the zero offset based on the Wang-Pan method when we obtain the IMF fluctuation events with a highly Alfvénic nature. Therefore, finding such IMF fluctuation events automatically is the key to achieving the automatic calculation of the zero offset. Here, we present an automatic procedure for calculating the zero offset by using the IMF fluctuations based on the Wang-Pan method. This automatic procedure consists of three parts: (1) selecting potential Alfvénic fluctuation events, (2) obtaining the OOL for each event, and (3) calculating the zero offset.

The magnetic field data measured by the FGM onboard the VEX spacecraft were used to illustrate the implementation of our automatic procedure. The VEX spacecraft, launched on 9 November 2005, is a three-axis stabilized spacecraft (Titov et al., 2006). The Venus Express is the first European mission to Venus, and one of its main scientific objectives is to study the interaction of the solar wind with Venus (Zhang TL et al., 2006). The VEX FGM measures the magnetic field with a sampling rate of up to $128 \mathrm{~Hz}$, using two triaxial fluxgate sensors. The ambient natural magnetic field and the dynamic field generated by the spacecraft can be separated based on the dual-sensor configuration (Zhang TL et al., 2006; Pope et al., 2011). In this study, we used the $1 \mathrm{~Hz}$ data from the VEX FGM in the spacecraft coordinate system to calculate the zero offset. Except for the zero offset, the sensitivities and non-orthogonal angles of the FGM have been calibrated, so we refer to these data we used as partially calibrated data.

\subsection{Selection of the Potential Alfvénic Fluctuation Event}

The solar wind has abundant magnetic field fluctuations and structures, such as Alfvén waves (Li H et al., 2016; Wu DJ et al., 2016), mirror mode structures (Volwerk et al., 2021; Wang GQ et al., 2021a), and discontinuities (Artemyev et al., 2019; Neukirch et al., 2020). We needed to select the magnetic field fluctuation with a highly Alfvénic nature from the partially calibrated data.

Figure 2 shows the partially calibrated magnetic field data in the spacecraft coordinate system between 00:00 UT on 1 January 2007 and 12:00 UT on 2 January 2007. According to the bow shock model (Shan LC et al., 2015) and the location of the VEX spacecraft, the VEX spacecraft was confirmed to be in the solar wind, as shown in the gray area of Figure 2. The magnetic field fluctuations have the following characteristics: (1) they do not have a fixed period, and the periods of the fluctuations vary from a few to hundreds of seconds; (2) the amplitude is dominant in different components of the magnetic field during different intervals; and (3) after removing the zero offset, the transverse component of the magnetic field fluctuations dominates in some intervals, whereas the compressional component dominates in other intervals.

The selection of the potential Alfvénic fluctuation event can be divided into two steps: (1) selection of the start and end moments of the event, and (2) evaluation of the Alfvénic nature of the event. Because the IMF has strong variations with periods typically less than 5 minutes, as shown in Figure 2, we only selected the IMF fluctuation events with periods within 5 minutes. To find the start and end times of a fluctuation event, the following procedures were executed in parallel on the three components of the magnetic field data:

(i) To reduce the effect of high-frequency noises, the $10 \mathrm{~s}$ boxcar filter was used to smooth the magnetic field data, and the result is marked as $B_{i \_s m 1}$ (the index $i$ represents the component $X, Y$, or $Z$ of the magnetic field in this procedure). To obtain the ambient magnetic field, the $300 \mathrm{~s}$ boxcar filter was used to smooth the magnetic field data, and the result was marked as $B_{i_{-} \text {sm2 }}$.

(ii) We obtained all the moments when the value of $B_{i \_s m} 1$ $-B_{i_{-} \text {sm2 }}$ was 0 , and the collection of these moments was marked as $T_{i}$. The first moment in $T_{i}$, marked as $T_{i} 0$, was regarded as the start time of the fluctuation event. The end moment $T_{i_{1}}$ of this fluctuation event was also in the collection of $T_{i}$ determined according to the following criteria: (a) $30 \mathrm{~s}<T_{i_{-} 1}-T_{i_{-} 0}<10 \mathrm{~min}$, and (b) the number of the elements in $T_{i}$ was in the range of 2 to 5 . When the above two criteria were met at the same time, the number of $T_{i}$ would be as large as possible.

(iii) The standard deviation $\delta_{i}$ of each magnetic field component was calculated in the period from $T_{i_{-} 0}$ to $T_{i_{-} 1}$.

(iv) From the above steps, we could obtain three periods. The period corresponding to the maximum standard deviation $\delta_{i}$ was determined as the eventual period of the fluctuation event.

(v) The end time of this event was selected as the start time of the next event. We repeated steps (i) to (iv) until we obtained the start and end times of all the fluctuation events in the solar wind in each VEX orbit.

According to the Wang-Pan method (Wang GQ and Pan ZH, 2021a) introduced in Section 2, we first built an offset cube in the same coordinate system as $\boldsymbol{B}_{M}$, and the three axes of the offset 


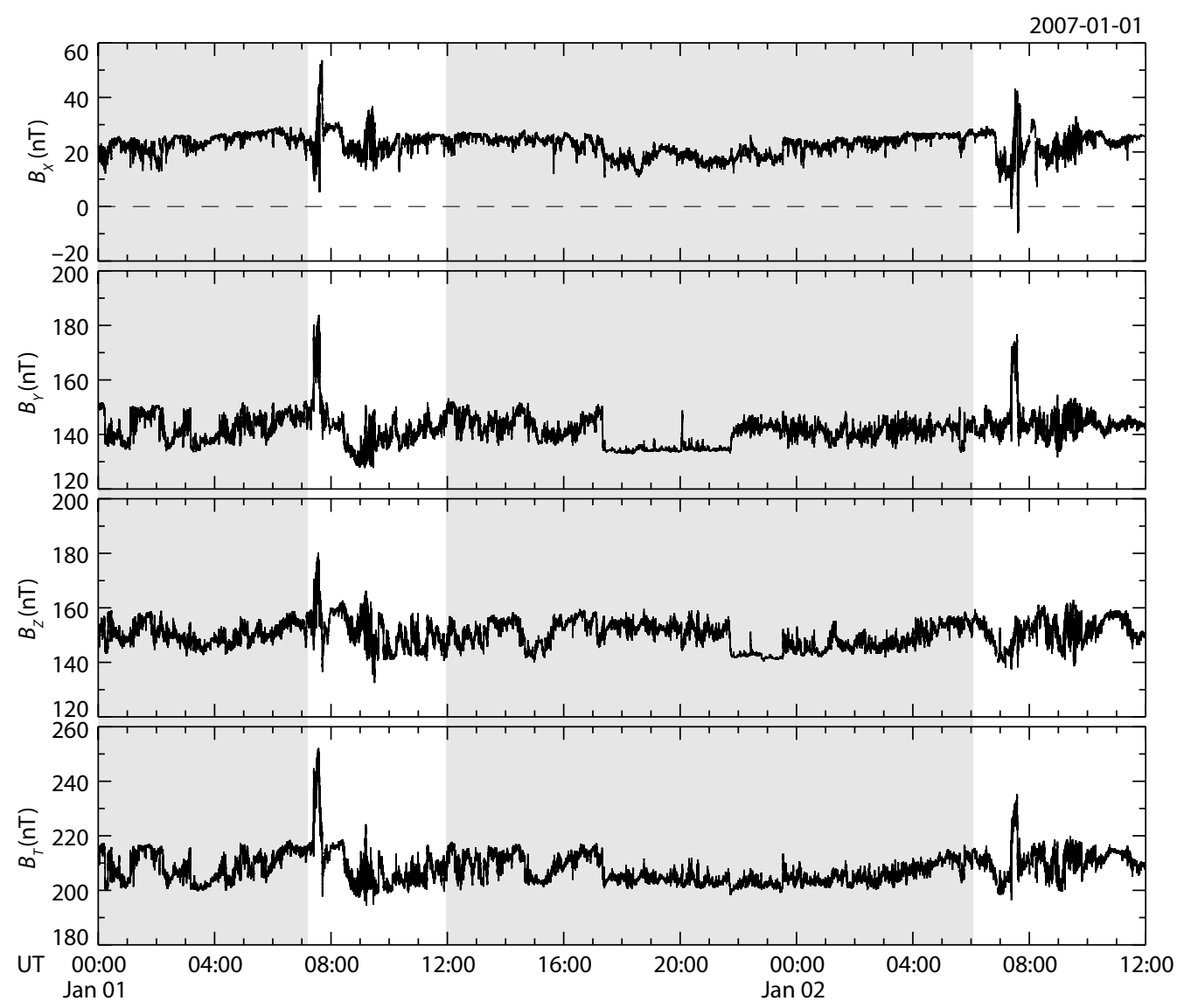

Figure 2. Partially calibrated magnetic field data of the VEX in the spacecraft coordinate system and its strength between 00:00 UT on 1 January 2007 and 12:00 UT on 2 January 2007. The gray areas indicate that the VEX spacecraft is in the solar wind.

cube were in the range of $\left(\left\langle B_{A_{-} X}\right\rangle-20,\left\langle B_{A_{-} X}\right\rangle+20\right),\left(\left\langle B_{A_{-} Y}\right\rangle-20\right.$, $\left.\left\langle B_{A} Y\right\rangle+20\right)$, and $\left(\left\langle B_{A} Z\right\rangle-20,\left\langle B_{A} Z\right\rangle+20\right) n \mathrm{nT}$, respectively. At point $\boldsymbol{O}^{\prime}$ in the offset cube, the magnetic field was modified as $\boldsymbol{B}_{M}^{\prime}=\boldsymbol{B}_{M}-$ $O^{\prime}$. We calculated the values of $\delta_{B_{T}^{\prime}}$ in the offset cube with a step length of $0.1 \mathrm{nT}$ along each axis. For a fluctuation event with a highly Alfvénic nature, the standard deviation of the total field strength was generally very small. Thus, the standard deviation $\delta_{B_{T}^{\prime}}$ of $\left|\boldsymbol{B}_{M}^{\prime}\right|$ was expected to be very small at a certain point in the offset cube when the real natural magnetic field $\boldsymbol{B}_{A}$ was a fluctuation event with a highly Alfvénic nature. If the minimum value of $\delta_{B_{T}^{\prime}}$ was $\left\langle\xi_{1}\right.$ (here, $\xi_{1}$ was set to be $0.1 \mathrm{nT}$ ) in the offset cube, this event had a high probability of being a highly Alfvénic fluctuation event. Thus, we identified this fluctuation event as a potential Alfvénic fluctuation event.

Figure 3 shows an example of the fluctuation event selected by using the partially calibrated data of the VEX spacecraft between 04:00 and 05:00 UT on 1 January 2007. The red curves indicate the three components of the ambient magnetic field. The gray areas in Figure 3 indicate the automatically selected potential Alfvénic fluctuation events with different periods. One can see that through the above procedures, we could obtain the interval of a fluctuation event with different temporal scales.

\subsection{Obtaining the OOL}

After obtaining the potential Alfvénic fluctuation events, we needed to determine the OOLs of these events. Note that these selected fluctuation events were likely to be fluctuation events with a highly Alfvénic nature, that is, we could not ensure thus far that all the selected events were highly Alfvénic fluctuations. The $\mathrm{OOL}$ was expected to be a straight line for a pure Alfvén wave (Wang GQ and Pan ZH, 2021a); thus, we needed to further identify whether these fluctuation events were highly Alfvénic by evaluating their OOLs. The OOL is usually not a straight line even for a highly Alfvénic fluctuation event because of the effect of the compressional fluctuation (Wang GQ and Pan ZH, 2021a). In addition, an OOL with a high linearity cannot be obtained if the normal direction of the plane is not selected properly for some events. Because we did not know in advance which axis would be the best choice to become the normal direction of the reference plane to obtain the minimum $\delta_{B_{T}^{\prime}}$, we used the following steps to obtain and evaluate the OOL:

(i) We found the points of $P_{O X}\left(=\left(P_{O X_{-} X_{1}} P_{O X_{-} Y_{1}} P_{O X_{-} Z}\right)\right)$, which are the minima of $\delta_{B_{T}^{\prime}}$ in the planes perpendicular to the $O_{x}^{\prime}$ axis with a step of $\Delta O_{X}^{\prime}=1 \mathrm{nT}$. Note that the $P_{O X}$ should not be located at the boundary of the plane. We required the number of $P_{O X}$ to be no less than 10. We then calculated the correlation coefficients between $P_{O X_{-} X}$ and $P_{O X_{-} Y}, P_{O X_{-} X}$ and $P_{O X_{-} Z}$, and $P_{O X_{-} Y}$ and $P_{O X_{-} Z}$ respectively. The maximum absolute value among these coefficients was noted as the $R_{O X}$. Similarly, we could obtain the sets of points $P_{O Y}$ and $P_{O Z}$ and their corresponding correlation coefficients $R_{O Y}$ and $R_{O Z}$. 


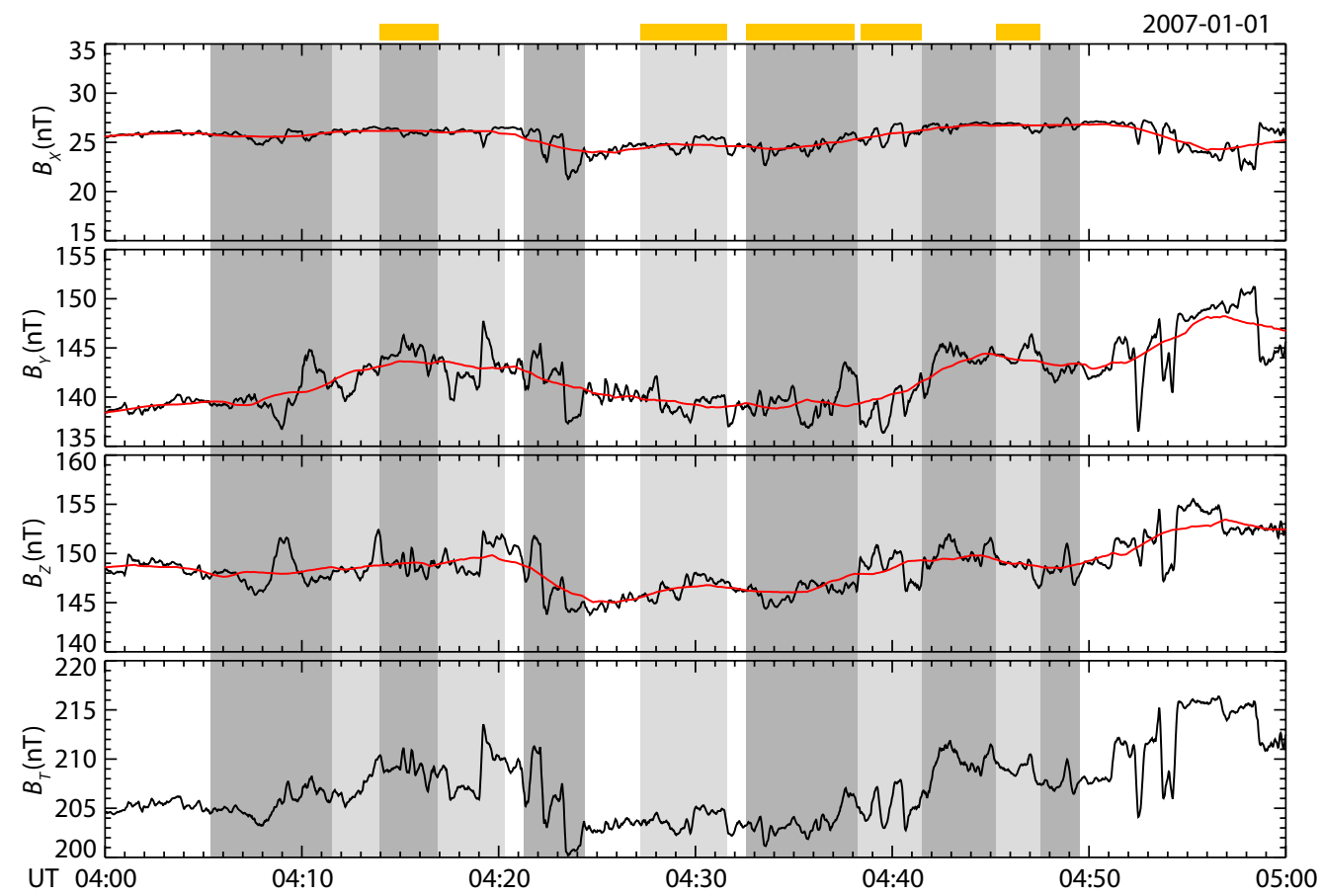

Figure 3. Partially calibrated magnetic field data of the VEX between 04:00 and 05:00 UT on 1 January 2007. The red curve denotes the ambient

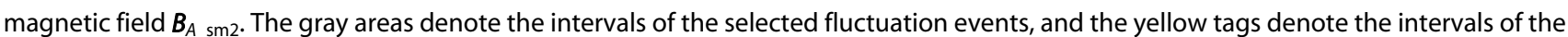
selected potential Alfvénic fluctuation events.

(ii) If $R_{O X}$ was larger than $R_{O Y}$ and $R_{O Z}$ and $R_{O X}$ was greater than $r$ (here, $r=0.9$ ), then $P_{O X}$ was selected to be the OOL. Similarly, $P_{O Y}$ or $P_{O Z}$ could be selected as the OOL when the $R_{O Y}$ or $R_{O Z}$ was the maximum of the three correlation coefficients and was greater than $r$. If $R_{O X}, R_{O Y}$, and $R_{O Z}$ were all $<0.9$, the corresponding event would not be selected to calculate the zero offset.

(iii) We then obtained the fitted straight line (marked as the FOOL) of the OOL determined in step (ii).

(iv) Steps (i) to (iv) were repeated until we obtained the FOOLs of all the selected highly Alfvénic fluctuation events that met the requirements in steps (i) and (ii).

Figure 3 displays 11 potential Alfvénic fluctuation events, as shown by the gray areas. We used the above procedure to further select the events whose OOLs had good linearity. As illustrated by the yellow tags in Figure 3, only 5 out of 11 events met the above criteria.

\subsection{Calculation of the Zero Offset}

The FOOL was expected to be parallel to the ambient magnetic field of $B_{A}$ and to pass through the zero offset in the offset cube for a pure Alfvén wave (Wang GQ and Pan ZH, 2021a). Because of the effect of compressional fluctuations, the FOOL does not pass through the zero offset. Therefore, Wang GQ and Pan ZH (2021a) optimized the zero offset so that the sum of the distances from the point to all the FOOLs was the smallest. We used the following steps to determine the zero offset:

(i) We selected $N_{F}$ adjacent FOOLs to determine the zero offset. Here, the number $N_{F}$ was set to be 16 . We required that all these FOOLs were within 1 day. (ii) We obtained the distance $L$ from the point $O^{\prime}$ to the FOOL in the offset cube. To reduce the influence of a certain FOOL deviating far from the estimated zero offset, we converted the distance $L$ to be a probability $f(L)$, where $f(L)=\frac{1}{\sqrt{2 \pi} \delta} \exp \left(-\frac{L^{2}}{2 \delta^{2}}\right)$. Here, we set the standard deviation as $\delta=3 \mathrm{nT}$. We determined the zero offset to be the point in the offset cube so that the sum of the values of $f(L)$ resulting from all the FOOLs was the largest. The average moments of these FOOLs were considered the moment of the estimated zero offset.

(iii) We used any $N_{F}-1$ out of the $N_{F}$ FOOLs to determine the zero offset by following the method described in step (ii). We could then obtain $N_{F}$ estimated zero offsets. The maximum and minimum of these $N_{F}$ zero offsets could be used to evaluate the calculation error of the zero offset.

(iv) We repeated steps (i) to (iii) to determine the zero offset of the next $N_{F}$ FOOLs whose sequence number was shifted by $M_{F}$ until all the FOOLs had been used to determine the zero offset. Here, $M_{F}$ was set to be 1 .

Figure 4 shows an example of the calculation of the zero offset when using 16 highly Alfvénic fluctuation events observed by the VEX on 1 January 2007. Figure 4a shows the FOOLs of the 16 events as well as their time intervals. As shown by the dots, the linearity of the OOL was high for each event. The red triangle in Figure $4 \mathrm{~b}$ denotes the zero offset $O_{1}(=(16.88,142.73,151) \mathrm{nT})$ determined by the automatic procedure introduced in this section, and the blue dot denotes the zero offset $O_{2}$ determined by any 15 out of the 16 events. The $X, Y$, and $Z$ components of $O_{2}$ were in the ranges of [16.8, 17.11], [142.66, 142.83], and [150.87, 151.13] nT, 

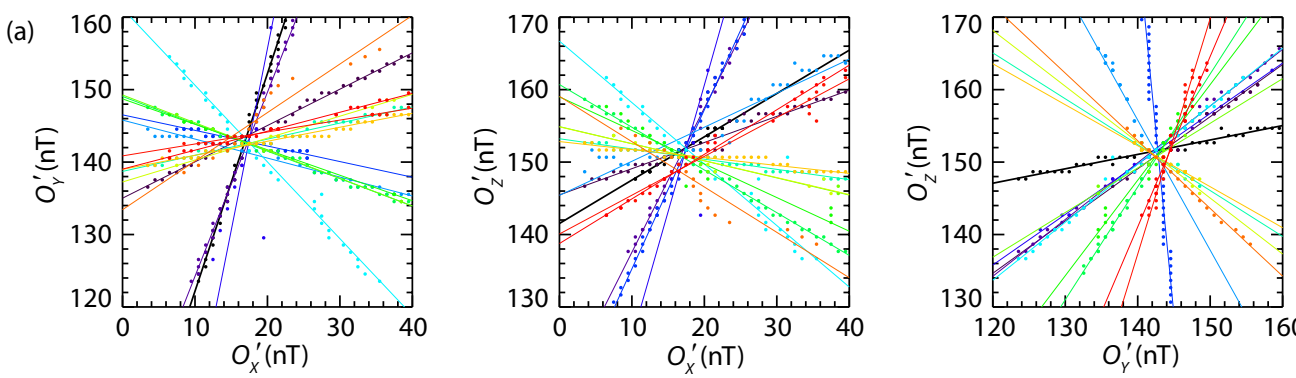

Time for each event 00:03:15-00:08:29 UT 01:18:03-01:23:40 UT 01:25:10-01:29:13 UT 01:29:13-01:32:41 UT 02:10:13-02:15:23 UT 03:06:27-03:14:50 UT 03:25:27-03:32:06 UT 04:13:57-04:16:54 UT 04:27:19-04:31:32 UT 04:32:32-04:38:17 UT
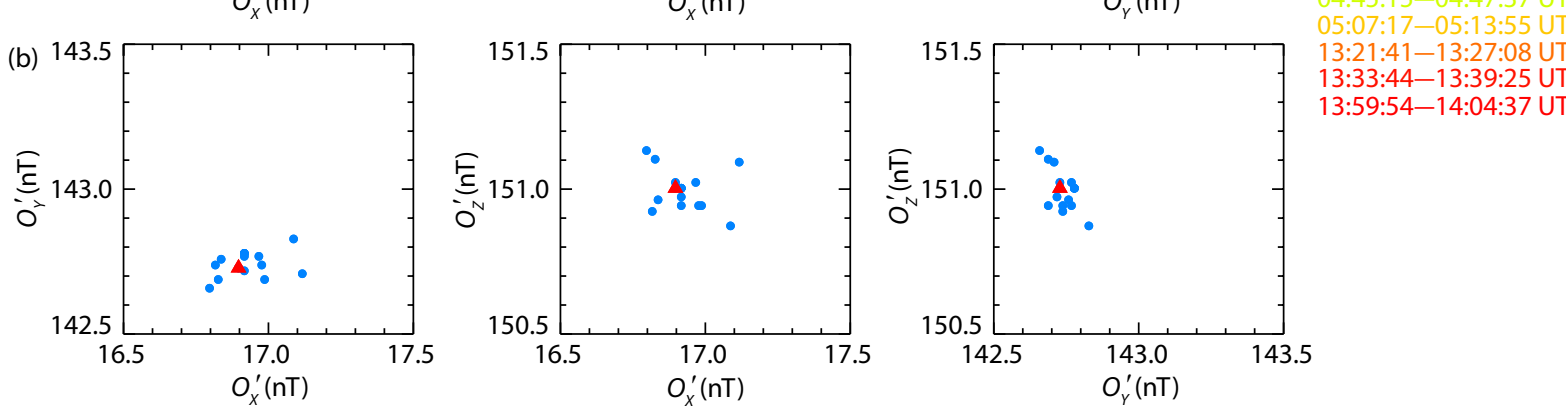

13:21:41-13:27:08 UT 13:33:44-13:39:25 UT 13:59:54-14:04:37 UT

Figure 4. (a) The FOOLs (solid lines) for the 16 highly Alfvénic fluctuation events observed by the VEX on 1 January 2007. Each dot denotes the position of the minimum $\delta_{B_{\tau}^{\prime}}$ in the corresponding plane for a certain event. The time intervals of the 16 events are also given. (b) The zero offset determined by the FOOLs. The red triangle denotes the zero offset determined by the 16 events. The blue dots denote the zero offset determined by any 15 out of 16 events.

respectively. The minimum and maximum of $\mathrm{O}_{2}$ can be used to evaluate the calculation error of $\boldsymbol{O}_{1}$.

\section{Application to the VEX}

We applied our automatic procedure to the partially calibrated data of the VEX from 1 January 2007 to 31 March 2007. According to the location of the VEX spacecraft and the model of the Venusian bow shock (Shan LC et al., 2015), we first found the time intervals during which the VEX spacecraft was in the solar wind. The data were then used to determine the zero offset based on the automatic procedure described in Section 3. The zero offsets determined by our procedure are shown by the red dots in Figure 5 . We also determined the zero offset by using the Davis-Smith method with the same fluctuation events, and the results are shown by the blue dots. For comparison, Figure 5 also displays the zero offset provided by the VEX FGM team, as shown by the orange triangles, and each day has one estimated zero offset. One can see that the profiles of the red, blue dots, and orange triangles are very similar, suggesting that our automatic procedure is successfully able to obtain reliable zero offsets. Figure 5 also shows that our automatic procedure can obtain several or more zero offsets within a day, which enables us to obtain variations of the zero offset. The zero offset consists of the instrumental offset and the static magnetic field of the spacecraft (Leinweber et al., 2008). The variations of the zero offset on a temporal scale of days (see the red circles in Figure 5) might be associated with the static magnetic field resulting from the spacecraft.

Figure 6 shows the difference between the zero offsets determined by the Wang-Pan method and the Davis-Smith method when using the same highly Alfvénic fluctuation events selected by our automatic procedure. $\Delta O$ is marked as the difference of the zero offsets determined by the two methods. Approximately $64.2 \%$ of the values of $\Delta O_{X}$ are within $[-0.5,0.5] \mathrm{nT}$, and the cor- responding probabilities of $\Delta O_{Y}$ and $\Delta O_{Z}$ are $87.2 \%$ and $73.9 \%$. This result suggests that the calculation results of the Wang-Pan method are very close to those of the Davis-Smith method when the same fluctuation events selected by our automatic procedure are used.

\section{Conclusion and Discussion}

To make the application of the Wang-Pan method more convenient, we developed a procedure to automatically find the fluctuation events with a highly Alfvénic nature in the solar wind and then determine the zero offset of the FGM. This automatic procedure consists of three parts: (1) selecting highly Alfvénic fluctuation events, (2) obtaining the OOL with a good linearity, and (3) determining the zero offset by using at least two nonparallel OOLs. We tested our automatic procedure by using 3 months of partially calibrated data measured by the VEX FGM and found that our automatic procedure was successful in achieving results as good as those from the Davis-Smith method.

Both the Wang-Pan method and the Davis-Smith method are based on the properties of Alfvén waves (Davis and Smith, 1968; Wang GQ and Pan ZH, 2021a); however, the solar wind has hardly any pure Alfvén waves (Leinweber et al., 2008). The compressional fluctuation can greatly affect the calculation accuracy of the zero offset by both methods (Leinweber et al., 2008; Wang GQ and Pan ZH, 2021a). Thus, the selection of fluctuation events with a highly Alfvénic nature is critical for both methods. Leinweber et al. (2008) provided the following three selection criteria for applying the Davis-Smith method: (1) the first criterion is designed to require the fluctuation in each data window to lie at least within a single plane; (2) the second criterion requires the magnetic field to have a low level of compression after being calibrated; (3) the third criterion requires each magnetic field component to have no strong correlation with the recalculated magnetic field strength. 


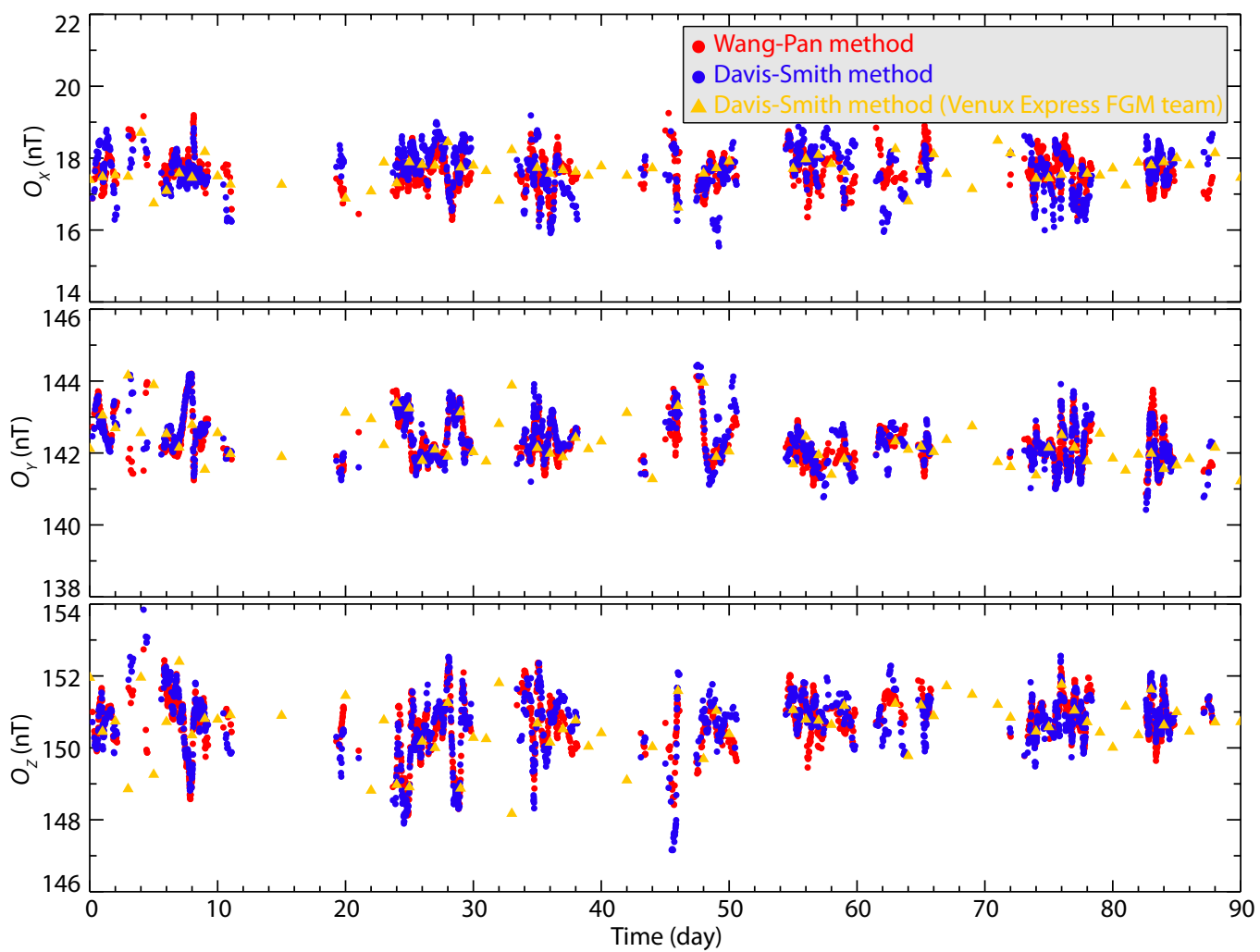

Figure 5. The zero offset for the VEX partially calibrated magnetic field data from 1 January 2007 to 31 March 2007. The red (blue) dots denote the zero offsets determined by the Wang-Pan (Davis-Smith) method. The orange triangles denote the zero offsets provided by the FGM team of the VEX spacecraft.

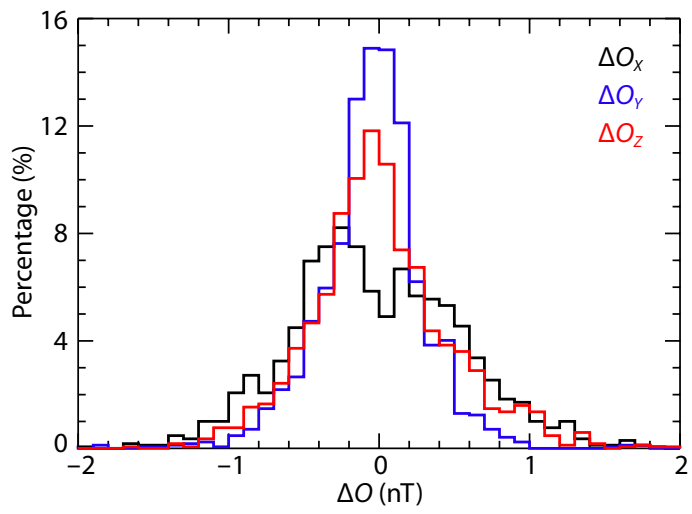

Figure 6. Distribution of the difference between the zero offsets determined by the Wang-Pan and the Davis-Smith method.

For the Wang-Pan method, we also needed to select the highly Alfvénic fluctuation events. The criteria for selecting highly Alfvénic fluctuation events can be summarized in the following two simple criteria: (1) the minimum of the standard deviations of the modified magnetic field strength in the offset cube must be small enough, such as less than $0.1 \mathrm{nT}$; (2) the OOL should have good linearity. One will find that these two selection criteria are more intuitive than those of the Davis-Smith method.

Our automatic procedure was developed based on the two criteria of the Wang-Pan method and consists of three parts: (1) selecting potentially Alfvénic fluctuation events, (2) obtaining the OOL with good linearity, and (3) determining the zero offset. The purpose of the first two parts is to select a highly Alfvénic fluctuation event. After the highly Alfvénic fluctuation event has been selected, we can choose either the Wang-Pan method or the Davis-Smith method to calculate the zero offset (Leinweber et al., 2008; Wang GQ and Pan ZH, 2021a). As shown in Figure 5, both methods produce very similar results. Therefore, our automatic procedure can also be used to automatically calculate the zero offset based on the Davis-Smith method. The parameters in our procedure, such as $\delta(=3 \mathrm{nT})$, are empirical values based on the VEX magnetic field data. If our automatic procedure were applied to the in-flight calibration of the FGM onboard other spacecraft, the parameters in our procedure could be modified so that our procedure could be better applied.

\section{Acknowledgements}

This work was financially supported by the Shenzhen Science and Technology Research Program (grant no. JCYJ20210324121412034) and the National Natural Science Foundation of China (grant nos. 41804157, 41774171, 41774167, 41974205, 41804157, and 41904156). The authors also acknowledge financial support from the pre-research Project on Civil Aerospace Technologies (grant no. D020103) funded by the China National Space Administration, the 111 Project (grant no. B18017), the Chinese Academy of Sciences Center for Excellence in Comparative Planetology, and the Macau Foundation. We thank Tielong Zhang for providing the magnetic field data from the Venus Express. 


\section{References}

Acuña, M. H. (2002). Space-based magnetometers. Rev. Sci. Instrum., 73(11), 3717-3736. https://doi.org/10.1063/1.1510570

Artemyev, A. V., Angelopoulos, V., Vasko, I. Y., Runov, A., Avanov, L. A., Giles, B. L., and Russell, C. T., and Strangeway, R. J. (2019). On the kinetic nature of solar wind discontinuities. Geophys. Res. Lett., 46(3), 1185-1194. https://doi.org/10.1029/2018gl079906

Balogh, A. (2010). Planetary magnetic field measurements: missions and instrumentation. Space Sci. Rev., 152(1-4), 23-97. https://doi.org/10.1007/s11214-010-9643-1

Belcher, J. W. (1973). A variation of the Davis-Smith method for in-flight determination of spacecraft magnetic fields. J. Geophys. Res., 78(28), 6480-6490. https://doi.org/10.1029/JA078i028p06480

Burch, J. L., Moore, T. E., Torbert, R. B., and Giles, B. L. (2016). Magnetospheric Multiscale overview and science objectives. Space Sci. Rev., 199(1-4), 5-21. https://doi.org/10.1007/s11214-015-0164-9

Davis, L., and Smith, E. J. (1968). The in-flight determination of spacecraft magnetic field zeros. Eos, Trans. Am. Geophys. Union, 49, 257.

Duan, A. Y., Zhang, H., and Lu, H. Y. (2018). 3D MHD simulation of the doublegradient instability of the magnetotail current sheet. Sci. China Technol. Sci., 61(9), 1364-1371. https://doi.org/10.1007/s11431-017-9158-7

Ge, Y. S., McFadden, J. P., Raeder, J., Angelopoulos, V., Larson, D., and Constantinescu, O. D. (2011). Case studies of mirror-mode structures observed by THEMIS in the near-Earth tail during substorms. J. Geophys. Res., 116(A1), A01209. https://doi.org/10.1029/2010ja015546

Hedgecock, P. C. (1975). A correlation technique for magnetometer zero level determination. Space Sci. Instrum., 1(1), 83-90.

Hellinger, P., Landi, S., Matteini, L., Verdini, A., and Franci, L. (2017). Mirror instability in the turbulent solar wind. Astrophys. J., 838(2), 158. https://doi.org/10.3847/1538-4357/aa67e0

Huang, S. Y., Sahraoui, F., Yuan, Z. G., Le Contel, O., Breuillard, H., He, J. S., Zhao, J. S., Fu, H. S., Zhou, M., ... Deng, X. H. (2018). Observations of whistler waves correlated with electron-scale coherent structures in the magnetosheath turbulent plasma. Astrophys. J., 861(1), 29. https://doi.org/10.3847/1538-4357/aac831

Keiling, A. (2009). Alfvén waves and their roles in the dynamics of the Earth's magnetotail: A review. Space Sci. Rev., 142(1-4), 73-156. https://doi.org/10.1007/s11214-008-9463-8

Leinweber, H. K., Russell, C. T., Torkar, K., Zhang, T. L., and Angelopoulos, V. (2008). An advanced approach to finding magnetometer zero levels in the interplanetary magnetic field. Meas. Sci. Technol., 19(5), 055104. https://doi.org/10.1088/0957-0233/19/5/055104

Li, H., Wang, C., Chao, J. K., and Hsieh, W. C. (2016). A new approach to identify interplanetary Alfvén waves and to obtain their frequency properties. J. Geophys. Res., 121(1), 42-55. https://doi.org/10.1002/2015ja021749

Liu, K., Hao, X. J., Li, Y. R., Zhang, T. L., Pan, Z. H., Chen, M. M., Hu, X. W., Li, X., Shen, C. L., and Wang, Y. M. (2020). Mars orbiter magnetometer of China's first Mars mission Tianwen-1. Earth Planet. Phys., 4(4), 384-389. https://doi.org/10.26464/epp2020058

Lu, S., Wang, R. S., Lu, Q. M., Angelopoulos, V., Nakamura, R., Artemyev, A. V., Pritchett, P. L., Liu, T. Z., Zhang, X. J., ... Wang, S. (2020). Magnetotail reconnection onset caused by electron kinetics with a strong external driver. Nat. Commun., 11(1), 5049. https://doi.org/10.1038/s41467-02018787-w

Meng, L. F., Pan, Z. H., Yi, Z., Wang, G. Q., and Zhang, T. L. (2018). Error properties of the fluxgate magnetometer offset based on Davis-Smith method. Chin. J. Geophys., 61(9), 3545-3551. https://doi.org/10.6038/ cjg2018L0264

Neukirch, T., Vasko, I. Y., Artemyev, A. V., and Allanson, O. (2020). Kinetic models of tangential discontinuities in the solar wind. Astrophys. J., 891(1), 86. https://doi.org/10.3847/1538-4357/ab7234

Olsen, N., Tøffner-Clausen, L., Sabaka, T. J., Brauer, P., Merayo, J. M. G., Jorgensen, J. L., Léger, J. M., Nielsen, O. V., Primdahl, F., and Risbo, T. (2003). Calibration of the Ørsted vector magnetometer. Earth Planets Space, 55(1), 11-18. https://doi.org/10.1186/Bf03352458
Pan, Z. H., Wang, G. Q., Meng, L. F., Yi, Z., and Zhang, T. L. (2019). Influence of Alfvénic characteristics on calibration of satellite magnetometer. Chin. J. Geophys., 62(4), 1193-1198. https://doi.org/10.6038/cjg2019M0513

Plaschke, F., and Narita, Y. (2016). On determining fluxgate magnetometer spin axis offsets from mirror mode observations. Ann. Geophys., 34(9), 759-766. https://doi.org/10.5194/angeo-34-759-2016

Plaschke, F., Goetz, C., Volwerk, M., Richter, I., Fruhauff, D., Narita, Y., Glassmeier, K. H., and Dougherty, M. K. (2017). Fluxgate magnetometer offset vector determination by the 3D mirror mode method. Monthly Notices of the Royal Astronomical Society, 469(Suppl_2), S675-S684. https://doi.org/10.1093/mnras/stx2532

Plaschke, F. (2019). How many solar wind data are sufficient for accurate fluxgate magnetometer offset determinations. Geosci. Instrum. Methods Data Syst., 8(2), 285-291. https://doi.org/10.5194/gi-8-285-2019

Pope, S. A., Zhang, T. L., Balikhin, M. A., Delva, M., Hvizdos, L., Kudela, K., and Dimmock, A. P. (2011). Exploring planetary magnetic environments using magnetically unclean spacecraft: a systems approach to VEX MAG data analysis. Ann. Geophys., 29(4), 639-647. https://doi.org/10.5194/angeo-29639-2011

Pudney, M. A., Carr, C. M., Schwartz, S. J., and Howarth, S. I. (2012). Automatic parameterization for magnetometer zero offset determination. Geosci. Instrum. Methods Data Syst., 1(2), 103-109. https://doi.org/10.5194/gi-1-1032012

Risbo, T., Brauer, P., Merayo, J. M. G., Nielsen, O. V., Petersen, J. R., Primdahl, F., and Richter, I. (2003). Ørsted pre-flight magnetometer calibration mission. Meas. Sci. Technol., 14(5), 674-688. https://doi.org/10.1088/09570233/14/5/319

Russell, C. T., Anderson, B. J., Baumjohann, W., Bromund, K. R., Dearborn, D., Fischer, D., Le, G., Leinweber, H. K., Leneman, D., ... Richter, I. (2016). The Magnetospheric Multiscale magnetometers. Space Sci. Rev., 199(1-4), 189-256. https://doi.org/10.1007/s11214-014-0057-3

Schmid, D., Plaschke, F., Narita, Y., Heyner, D., Mieth, J. Z. D., Anderson, B. J., Volwerk, M., Matsuoka, A., and Baumjohann, W. (2020). Magnetometer inflight offset accuracy for the BepiColombo spacecraft. Ann. Geophys., 38(4), 823-832. https://doi.org/10.5194/angeo-38-823-2020

Shan, L. C. , Lu, Q. M. , Mazelle, C. , Huang, C. , Zhang, T. L. , Wu, M. Y. , Gao, X. L. , and Wang, S. (2015). The shape of the Venusian bow shock at solar minimum and maximum: revisit based on VEX observations. Planet. Space Sci. , 109-110, 32-37. https://doi.org/10.1016/j.pss.2015.01.004

Sun, J. C., Gao, X. L., Ke, Y. G., Lu, Q. M., Wang, X. Y., and Wang, S. (2019). Expansion of solar coronal hot electrons in an inhomogeneous magnetic field: 1D PIC simulation. Astrophys. J., 887(1), 96. https://doi.org/10.3847/1538-4357/ab5060

Sun, J. C., Chen, L. J., and Wang, X. Y. (2020). Wave normal angle distribution of magnetosonic waves in the earth's magnetosphere: 2-D PIC simulation. J. Geophys. Res., 125(5), e2020JA028012. https://doi.org/10.1029/ 2020ja028012

Sun, J. C., Wang, G. Q., Zhang, T. L., Hu, H. Q., and Yang, H. G. (2022). Evidence of Alfvén waves generated by mode coupling in the magnetotail lobe. Geophys. Res. Lett., 49(1), e2021GL096359. https://doi.org/10.1029/2021GL096359

Titov, D. V., Svedhem, H., Koschny, D., Hoofs, R., Barabash, S., Bertaux, J. L., Drossart, P., Formisano, V., Häusler, B., ... Clochet, A. (2006). Venus Express science planning. Planet. Space Sci., 54(13-14), 1279-1297. https://doi.org/10.1016/j.pss.2006.04.017

Volwerk, M., Mautner, D., Wedlund, C. S., Goetz, C., Plaschke, F., Karlsson, T., Schmid, D., Rojas-Castillo, D., Roberts, O. W., and Varsani, A. (2021). Statistical study of linear magnetic hole structures near Earth. Ann. Geophys., 39(1), 239-253. https://doi.org/10.5194/angeo-39-239-2021

Wang, G. Q., Ge, Y. S., Zhang, T. L., Nakamura, R., Volwerk, M., Baumjohann, W., Du, A. M., and Lu, Q. M. (2015). A statistical analysis of Pi2-band waves in the plasma sheet and their relation to magnetospheric drivers. J. Geophys. Res., 120(8), 6167-6175. https://doi.org/10.1002/2014ja020753

Wang, G. Q., Zhang, T. L., and Volwerk, M. (2016). Statistical study on ultralowfrequency waves in the magnetotail lobe observed by Cluster. J. Geophys. Res., 121(6), 5319-5332. https://doi.org/10.1002/2016ja022533 
Wang, G. Q., Volwerk, M., Zhang, T. L., Schmid, D., and Yoshikawa, A. (2017). High-latitude $\mathrm{Pi} 2$ pulsations associated with kink-like neutral sheet oscillations. J. Geophys. Res., 122(3), 2889-2899. https://doi.org/10.1002/2016ja023370

Wang, G, Q., Pan, Z. H., Hu, X. W., Liu, K., Meng, L. F., Yi, Z., and Zhang, T. L. (2019). Numerical simulation of influence of compressional fluctuation amplitude on zero correction of satellite magnetometer. Spacecr. Environ. Eng., 36(3), 229-234. https://doi.org/10.12126/see.2019.03.005

Wang, G. Q., Volwerk, M., Xiao, S. D., Wu, M. Y., Hao, Y. F., Liu, L. J., Wang, G., Chen, Y. Q., and Zhang, T. L. (2020a). Three-dimensional geometry of the electron-scale magnetic hole in the solar wind. Astrophys. J. Lett., 904(1), L11. https://doi.org/10.3847/2041-8213/abc553

Wang, G. Q., Cheng, S. N., Meng, L. F., Yi, Z., Xiao, Q., Pan, Z. H., Hu, X. W., Liu, K., and Zhang, T. L. (2020b). Effect of reference coordinate system on the offset of fluxgate magnetometer based on Davis-Smith method. Spacecr. Environ. Eng., 37(6), 570-575. https://doi.org/10.12126/see.2020.06.006

Wang, G. Q., and Pan, Z. H. (2021a). A new method to calculate the fluxgate magnetometer offset in the interplanetary magnetic field: 1. Using Alfvén waves. J. Geophys. Res., 126(4), e2020JA028893. https://doi.org/10.1029/2020ja028893

Wang, G. Q., Volwerk, M., Wu, M. Y., Hao, Y. F., Xiao, S. D., Wang, G., Liu, L. J., Chen, Y. Q., and Zhang, T. L. (2021a). First observations of an ion vortex in a magnetic hole in the solar wind by MMS. Astron. J., 161(3), 110. https://doi.org/10.3847/1538-3881/abd632

Wang, G. Q., and Pan, Z. H. (2021b). A new method to calculate the fluxgate magnetometer offset in the interplanetary magnetic field: 2. Using mirror mode structures. J. Geophys. Res., 126(9), e2021JA029781. https://doi.org/10.1029/2021ja029781

Wang, G. Q., Zhang, T. L., Wu, M. Y., Xiao, S. D., Wang, G., Chen, Y. Q., Sun, J. C., and Volwerk, M. (2021b). Field-aligned currents originating from the chaotic motion of electrons in the tilted current sheet: MMS observations. Geophys. Res. Lett., 48(9), e2020GL088841. https://doi.org/10.1029/ 2020gl088841

Wu, D. J., Feng, H. Q., Li, B., and He, J. S. (2016). Nature of turbulence, dissipation, and heating in space plasmas: from Alfvén waves to kinetic Alfvén waves. J. Geophys. Res., 121(8), 7349-7352. https://doi.org/10.1002/2016ja023082

Xiao, S. D., Wu, M. Y., Wang, G. Q., Wang, G., Chen, Y. Q., and Zhang, T. L. (2020a). Turbulence in the near-Venusian space: Venus express observations. Earth Planet. Phys., 4(1), 82-87. https://doi.org/10.26464/ epp2020012

Xiao, S. D., Zhang, T. L., Vörös, Z., Wu, M. Y., Wang, G. Q., and Chen, Y. Q. (2020b). Turbulence near the Venusian bow shock: Venus Express observations. J. Geophys. Res., 125(2), e2019JA027190. https://doi.org/10.1029/ 2019JA027190

Zhang, T. L., Baumjohann, W., Delva, M., Auster, H. U., Balogh, A., Russell, C. T., Barabash, S., Balikhin, M., Berghofer, G., ... Lebreton, J. P. (2006). Magnetic field investigation of the Venus plasma environment: expected new results from Venus Express. Planet. Space Sci., 54(13-14), 1336-1343. https://doi.org/10.1016/j.pss.2006.04.018

Zhang, T. L., Lu, Q. M., Baumjohann, W., Russell, C. T., Fedorov, A., Barabash, S., Coates, A. J., Du, A. M., Cao, J. B., ... Balikhin, M. (2012). Magnetic reconnection in the near Venusian magnetotail. Science, 336(6081), 567-570. https://doi.org/10.1126/science.1217013 